\title{
Eutectic System Based on Urea and Potassium Sodium Tartrate
}

\author{
Mae Joanne B. Aguila*, Joshua P. Gemperoso, King Bryan C. Gabog, Al Jerome A. Magsino \\ Institute of Chemistry, College of Arts and Sciences, University of the Philippines Los Baños, College, Laguna
}

\begin{abstract}
Deep eutectic solvents (DESs) are considered as better alternative solvents in chemical and physical processes. The binary mixture of urea and potassium sodium tartrate is explored in this study. A eutectic system is determined at composition made up of $33 \%$ potassium sodium tartrate and $67 \%$ urea (1:2 molar ratio). This eutectic system has a freezing point of $19.83 \pm 0.76{ }^{\circ} \mathrm{C}$, density of $1.1971 \pm 0.0003 \mathrm{~g} \mathrm{~mL}^{-1}$, and viscosity of $34.4226 \pm 0.0665 \mathrm{cP}$. The most stable conformation for the adduct of urea and potassium sodium tartrate with water molecules was determined through density functional calculations. The gas phase total energy for the adduct was determined as $-5576863 \mathrm{~kJ} \mathrm{~mol}^{-1}$. Electrostatic interactions between the cations and the carboxylate sites are present, H-bonding between protons of urea and the hydroxyl oxygen of the tartrate, and intramolecular H-bonding between the hydroxyl and carboxylate sites of tartrate are accounted for in the structure. The total energy associated with nonbonding interactions is computed as $-826 \mathrm{~kJ} \mathrm{~mol}^{-1}$, which suggests that these interactions stabilize the formation of the DES system of potassium sodium tartrate and urea.
\end{abstract}

Keywords: deep eutectic solvent; hydrogen bonding; urea-tartrate adduct; DFT calculations

\section{INTRODUCTION}

In common physical and chemical process, solvent is used as common medium for reacting species, separating components of mixtures, purifying via extraction, and washing of substances. Unfortunately, most available solvents have the drawbacks of being harmful and toxic. Typical organic solvents are volatile, flammable, explosive, and have low biodegradability. With the increasing consciousness about green chemistry, more environmentally benign and less hazardous chemicals and methodologies are targeted (Clarke et al., 2018).

In the recent years, ionic liquids, low melting mixtures, and deep eutectic systems are being developed as solvent alternatives. Ionic liquids (ILs) are mixtures of organic salts, commonly made up of organic cation and organic or inorganic anion
(Zhang et al., 2006). Most cases are made up of asymmetrically substituted organic cation, such as ammonium or phosphonium, and halogenbased anions. Although ionic liquids have many advantageous properties over common organic solvents such as low volatility and high recyclability, most ILs have high toxicity.

Low melting mixtures (LMMs) and deep eutectic solvents (DESs) are mixtures of two components, one is considered as hydrogen bond donor (HBD) and the other as hydrogen bond acceptor (HBA) (Zhang et al., 2012; Liu et al., 2015; Khandelwal et al., 2016). DESs are generally classified as type I, II, III, and IV, depending on the types of molecules used as components. These solvents have the advantages of ease of preparation, low to negligible vapor pressure, wide liquid range, and good solvation properties. These solvents are considered 
to be a greener alternative over ionic liquids since the components are generally more biocompatible. Most DESs being reported in the literature are based on urea, glycerol, or sugar. The most commonly used DES choline chloride: urea (1:2) has been applied to various applications including metal extraction, $\mathrm{CO}_{2}$ sequestration, and catalysis medium.

Here, we report the preparation of a low melting binary mixture of urea and potassium sodium tartrate. These chemicals have no or low toxicity, are readily available being biorenewable, and cheap. The low melting binary mixture of these two components may be a suitable alternative for solvents at ambient temperatures.

\section{EXPERIMENTAL}

Materials. Urea and potassium sodium $\mathrm{L}-(+)-$ tartrate tetrahydrate (KNa tartrate) were obtained from Ajax Finechem at $99.00 \%$ purity and were used as received without further purification. The properties of these two components are summarized in Table 1.

Table 1. Properties of Components Used in This Work (Lide, 2005).

\begin{tabular}{llll}
\hline $\begin{array}{l}\text { Com- } \\
\text { pound }\end{array}$ & Molecular Formula & $\begin{array}{l}\text { Molar } \\
\text { Mass, g } \\
\text { mol-1 }^{-1}\end{array}$ & $\begin{array}{l}\text { Melting } \\
\text { Point, }\end{array}$ \\
\hline Urea & $\mathrm{CH}_{4} \mathrm{~N}_{2} \mathrm{O}$ & 60.06 & 133.3 \\
\hline $\mathrm{NaK}$ & $\mathrm{NaKC}_{4} \mathrm{H}_{4} \mathrm{O}_{6} \bullet 4 \mathrm{H}_{2} \mathrm{O}$ & 282.22 & 70.0 \\
Tartrate & & & \\
\hline
\end{tabular}

Methodology. Binary Solid-Liquid Phase Diagram. The cooling curves for the mixtures were measured with a fabricated apparatus consisted of a $20-\mathrm{mL}$ test tube with rubber stopper fitted with a stirrer and thermometer, and immersed in Dewar flask filled with crushed ice (Gallus et al., 2001). With constant amount of urea $(2.00 \mathrm{~g}, 0.0333 \mathrm{~mol})$, binary mixtures of urea and $\mathrm{KNa}$ tartrate were prepared by varying the amount of $\mathrm{KNa}$ tartrate to arrive at mole ratios of urea with $\mathrm{KNa}$ tartrate as follows $\chi_{\text {urea }}$ $=0.25,0.33,0.50,0.55,0.60,0.67,0.70,0.75$. The compounds were ground using a mortar and pestle until the mixture liquefied or until a smooth paste was formed. These liquefied mixtures were sonicated for $15 \mathrm{~min}$ and heated at $80-100{ }^{\circ} \mathrm{C}$ until homogeneity. The liquefied mixture was placed in the cooling curve apparatus and the temperature of cooling was recorded every 10s until temperature reached $10{ }^{\circ} \mathrm{C}$ below the measured detected arrest temperature. Break and arrest temperatures were determined from the cooling curves (temperature versus time) (Williams and Collins, 1994; Deacutis et al., 1997). Each measurement was done in triplicate and standard deviation was reported.

Density and Viscosity Measurements. The $\mathrm{KNa}$ tartrate: urea (1:2) solvent was prepared as described above, and stored in silica desiccator for overnight prior to measurements. The density of $\mathrm{KNa}$ tartrate: urea (1:2) system was determined at $28{ }^{\circ} \mathrm{C}$ using the pycnometer method (Garland et al., 2009), by comparing the mass ratios between the DES and the calibration liquid (water), using the formula:

$$
\rho_{s u b s}=\frac{m_{p y c+s u b s}-m_{p y c}}{m_{p y c+w a t e r}-m_{p y c}} \times \rho_{\text {water at RT }}
$$

Each measurement was done in triplicate and standard deviation was reported.

The kinematic viscosity of the synthesized $\mathrm{KNa}$ tartrate: urea (1:2) systen was determined using a Cannon-Fenske viscometer through the capillary flow method (Garland et al., 2009). Capillary dimensions ( $\mathrm{A}$ and $\mathrm{B}$ ) were determined prior to analysis, using different calibrating liquids - water, ethyl acetate, acetone, through the Hagen-Poiseuille equation:

$$
\eta=A p t-\frac{B p}{t}, \text { where } A=\frac{\pi r^{4} g h}{8 V l} \text { and } B=\frac{V}{8 \pi l}
$$

The viscosity was then determined by measuring the flowtime of the solution in the calibrated CannonFenske viscometer at $28{ }^{\circ} \mathrm{C}$. Each measurement was done in triplicate and standard deviation was reported.

Density Functional Theory (DFT) Studies. All programs used were open software accessed on 07 June 2018 through the websites: GAMESS - http:// www.msg.ameslab.gov/gamess/, Avogadro - https:// avogadro.cc, and MacMolPlt - https://brettbode. github.io/wxmacmolplt/.

Structures were constructed using the Avogadro software (Hanwell et al., 2012). The most probable conformation in the gas phase of components in the DES system was constructed from geometryoptimized urea, $\mathrm{KNa}$ tartrate, and water molecules through the GAMESS program (Schmidt et al., 1993) using the B3LYP hybrid functional with 6-31G(d) basis set. Visualization of the hydrogen bonding interactions was done using the MacMolPlt program (Bode and Gordon, 1998). All calculations were done in the gas phase and at $298.15 \mathrm{~K}$. The total energy associated with the nonbonding interactions in the DES systems $\left(\Delta \mathrm{E}_{\mathrm{NB}}\right)$ was calculated using the equation:

$$
\Delta E_{\mathrm{NB}}=\Delta E_{\mathrm{DES}}-\Delta E_{\mathrm{KNaTartrate}}-2 \times \Delta E_{\text {urea }}-4 \times \Delta E_{\mathrm{H}_{2} \mathrm{O}}
$$


where $\Delta \mathrm{E}_{\mathrm{DES}}, \Delta \mathrm{E}_{\mathrm{KNa} \text { Tarrtrate }}, \Delta \mathrm{E}_{\text {urea }}, \Delta \mathrm{E}_{\mathrm{H} 2 \mathrm{O}}$ are the total energies of the urea: $\mathrm{KNa}$ tartrate $(1: 2)$ system, $\mathrm{KNa}$ tartrate, urea, and water, respectively (Hao, 2006).

\section{RESULTS AND DISCUSSIONS}

The binary mixtures of urea and $\mathrm{KNa}$ tartrate were prepared via grinding, sonication and heating. Grinding is necessary to prevent decomposition of the individual components, forming a paste. After sonication and heating, we achieved homogenous, transparent binary systems; but only the binary mixture of mole fraction $\chi_{\text {urea }}=0.67$ remained in clear liquid form after cooling to room temperature $\left(-30^{\circ} \mathrm{C}\right)$, while the rest have solidified.

The solid-liquid phase diagram for the binary mixtures of $\mathrm{KNa}$ tartrate: urea is shown in Figure 1. The eutectic composition occurs at the molar ratio of $1: 2$ for $\mathrm{KNa}$ tartrate: urea $\left(\chi_{\text {urea }}=0.67\right)$, with a freezing point of $19.83 \pm 0.76^{\circ} \mathrm{C}$, Table 2 . This temperature is significantly lower than freezing points of the pure components, allowing for an ambient temperature solvent mixture. The freezing temperature of the eutectic solvent is comparable to that of choline chloride: urea (1:2) system (at $\left.12{ }^{\circ} \mathrm{C}\right)$, but lower than that of for choline chloride: L-(+)-tartaric acid (at $47^{\circ} \mathrm{C}$ ) (Abbott et al., 2003; Shahbaz et al., 2010; Hayyan et al., 2010; Maugeri and Dominguez de Maria, 2012). A related DES was used by Gore et al. (2011) made from L-(+)tartaric acid: dimethylurea (30:70). Although the physical properties of this DES were not determined at that time, this DES was prepared as melt at 70 ${ }^{\circ} \mathrm{C}$ and was used a medium for catalysis at that temperature (Gore et al., 2011; Krishnakumar et al., 2014). Generally, DESs which are liquid at room temperature, thus have freezing point lower than $50^{\circ} \mathrm{C}$, are promising solvents for many applications (Khandelwal et al., 2016).

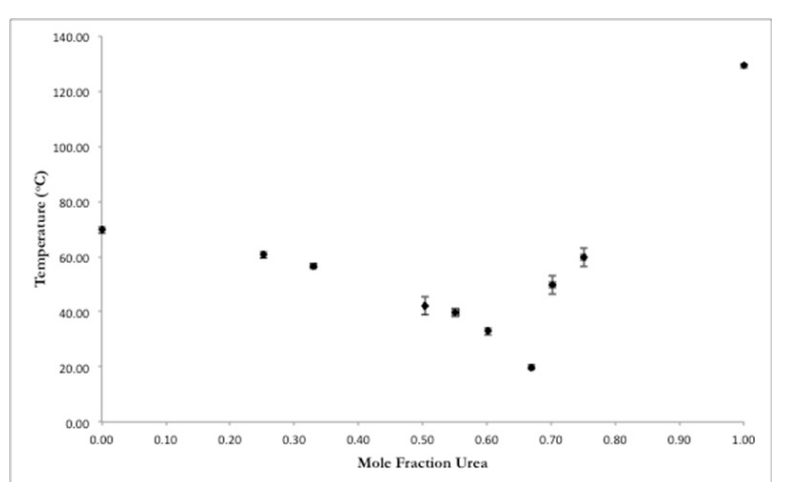

Figure 1. Freezing Point for the Binary Mixture of Urea and KNa tartrate as a Function of Composition.

The density and viscosity of the $\mathrm{KNa}$ tartrate: urea (1:2) eutectic system are determined as $1.1971 \pm$ $0.0003 \mathrm{~g} \mathrm{~cm}^{-1}$ and $34.4226 \pm 0.0665 \mathrm{cP}$ at $28^{\circ} \mathrm{C}$, respectively (Table 2). As with most DESs prepared, the eutectic system KNa tartrate: urea (1:2) is denser than water at the same temperature (Zhang et al., 2012). This $\mathrm{KNa}$ tartrate: urea (1:2) system is less dense than the choline chloride: urea and $\mathrm{ZnCl}_{2}$ : urea systems, but falls within similar density range with the choline chloride: polyol systems (glycerol and ethylene glycol) (Abbott et al., 2003; Abbott et al., 2011; Craveiro et al., 2016; Abbott and Barron et al., 2007; Abbott et al., 2006; D'Agostino et al., 2011; Abbott and Harris et al., 2007; Shahbaz et al., 2012). The eutectic system $\mathrm{KNa}$ tartrate: urea (1:2) is relatively more viscous than common molecular solvents (Zhang et al., 2012). It is less viscous than choline chloride: urea, choline chloride: glycerol, and $\mathrm{ZnCl}_{2}$ : urea systems, but comparable to the choline chloride: ethylene glycol system (Abbott $e t$ al., 2003; Abbott et al., 2011; Abbott and Barron et al., 2007; D'Agostino et al., 2011; Abbott and Harris et al., 2007). This low viscosity type of DES is desirable for applications as medium in chemical and physical processes (Zhang et al., 2012).

To gain further insights into the network of interactions between urea and $\mathrm{KNa}$ tartrate in the adduct, we performed DFT calculations using the B3LYP method (Hao, 2006; Zhang et al., 2014; Zhu et al., 2016; Palomba et al., 2018). We have included the cations, $\mathrm{Na}^{+}$and $\mathrm{K}^{+}$, as well as four water molecules in the structure optimization. The most stable gas configuration of the $\mathrm{KNa}$ tartrate: urea (1:2) adduct is shown in Figure 2. As shown, the cations are closer to the carboxylate sites, through electrostatic interaction. Each cation has a water molecule that surrounds it. These water molecules form hydrogen bonds with the carboxylate (O12-H17, O5-H15). The oxygens of one carboxylate moiety are also intramolecularly $\mathrm{H}$-bonded to the hydroxyls (O5$\mathrm{H} 8, \mathrm{O} 11-\mathrm{H} 10)$. The urea molecules form hydrogen bonds with the oxygen of the hydroxyl groups of the tartrate. Interestingly, the structure shows that the one urea molecule forms only one $\mathrm{H}$-bond end (O6-H36), whereas two hydrogens of the second urea molecule form $\mathrm{H}$-bonds to the same oxygen site (O9-H27, O9-H38). Because of this, the former is shorter $(1.890 \AA)$ than the latter $(2.020 \&$ $2.103 \AA$ ) or with the mean experimental N-H---O hydrogen bonds (1.921(4) ^) (Taylor et al., 1984). The remaining water molecules are both $\mathrm{H}$-bonded intermolecularly to the second urea molecule though carbonyl O of urea, $\mathrm{O} 25-\mathrm{H} 18$, and protonic hydrogen, O23-H39. These water molecules seem to lock this weakly $\mathrm{H}$-bonded urea to the tartrate better by acting as the mediating species, one water molecule is bonded to both the protonic hydrogen of urea and the oxygen of the carboxylate group of the tartrate. This water molecule is also $\mathrm{H}$-bonded to the other water molecule that is bonded to the carbonyl 
Table 2. Main Physical Properties of Water, Common Organic Solvents, and DESs.

\begin{tabular}{|c|c|c|c|c|}
\hline Solvent (mol ratio) & $\begin{array}{l}\text { Freezing point } \\
\qquad \mathrm{T}_{\mathrm{f}},{ }^{\circ} \mathrm{C}\end{array}$ & $\begin{array}{l}\text { Density } \rho, g \mathrm{~mL}^{-1} \\
\quad(\mathrm{Temp})\end{array}$ & $\begin{array}{l}\text { Viscosity } \eta, c P \\
\quad(\text { Temp) }\end{array}$ & Literature \\
\hline Water & 0 & $0.997\left(25^{\circ} \mathrm{C}\right)$ & $0.835052\left(28^{\circ} \mathrm{C}\right)$ & $\begin{array}{l}\text { (Lide, 2005; Dormund Data Bank, acceessed } \\
\text { June 21, 2018) }\end{array}$ \\
\hline Ethyl acetate & -83.8 & $0.9003\left(20^{\circ} \mathrm{C}\right)$ & $0.416921\left(28^{\circ} \mathrm{C}\right)$ & $\begin{array}{l}\text { (Lide, 2005; Dormund Data Bank, acceessed } \\
\text { June 21, 2018) }\end{array}$ \\
\hline Acetone & -94.7 & $0.7845\left(25^{\circ} \mathrm{C}\right)$ & $0.300479\left(28^{\circ} \mathrm{C}\right)$ & $\begin{array}{l}\text { (Lide, 2005; Dormund Data Bank, acceessed } \\
\text { June 21, 2018) }\end{array}$ \\
\hline $\begin{array}{l}\text { Choline Chloride: } \\
\text { Urea }(1: 2)\end{array}$ & 12 & $1.25\left(25^{\circ} \mathrm{C}\right)$ & $750\left(25^{\circ} \mathrm{C}\right)$ & $\begin{array}{l}\text { (Abbott et al., 2003; Abbott et al., 2006; } \\
\text { D'Agostino et al., 2011) }\end{array}$ \\
\hline $\begin{array}{l}\text { Choline Chloride: } \\
\text { Glycerol }(1: 2)\end{array}$ & -40 & $1.18\left(25^{\circ} \mathrm{C}\right)$ & $376\left(25^{\circ} \mathrm{C}\right)$ & $\begin{array}{l}\text { (Hayyan et al., 2010; Abbott et al., 2011; } \\
\text { Abbott and Harris et al., 2007) }\end{array}$ \\
\hline $\begin{array}{l}\text { Choline Chloride: } \\
\text { Ethylene Glycol } \\
(1: 2)\end{array}$ & -66 & $1.12\left(25^{\circ} \mathrm{C}\right)$ & $37\left(25^{\circ} \mathrm{C}\right)$ & $\begin{array}{l}\text { (Shahbaz et al., 2012; D'Agostino et al., } \\
\text { 2011; Abbott and Harris et al., 2007; Shah- } \\
\text { baz et al., 2012) }\end{array}$ \\
\hline $\begin{array}{l}\text { Choline Chloride: } \\
\text { L-(+)-Tartaric Acid } \\
(1: 0.5)\end{array}$ & 47 & $1.26\left(23^{\circ} \mathrm{C}\right)$ & - & $\begin{array}{l}\text { (Craveiro et al., 2016; Maugeri and Domin- } \\
\text { guez de Maria, 2012) }\end{array}$ \\
\hline $\mathrm{ZnCl}_{2}:$ Urea $(1: 3.5)$ & 9 & $1.63\left(25^{\circ} \mathrm{C}\right)$ & $11340\left(25^{\circ} \mathrm{C}\right)$ & $\begin{array}{l}\text { (Abbott and Barron et al., 2007; Abbott and } \\
\text { Harris et al., 2007) }\end{array}$ \\
\hline $\begin{array}{l}\text { KNa Tartrate: Urea } \\
(1: 2)\end{array}$ & $19.83 \pm 0.76$ & $\begin{array}{l}1.1971 \pm 0.0003 \\
\left(28^{\circ} \mathrm{C}\right)\end{array}$ & $\begin{array}{l}34.4226 \pm 0.0665 \\
\left(28^{\circ} \mathrm{C}\right)\end{array}$ & This work \\
\hline
\end{tabular}

group of urea. Moreover, the computed total energy of the DES system is lower in comparison to the sum of the total energies of individual equilibrium geometries of urea, $\mathrm{KNa}$ tartrate, and water. With a $\Delta \mathrm{E}_{\mathrm{NB}}$ value of $-826 \mathrm{~kJ} \mathrm{~mol}^{-1}$ (Table 3), this network of non-covalent interactions contributes to the stability of the DES formation.

Table 3. Comparison of the Calculated Total Energies for the KNa tartrate:urea (1:2) DES with the Individual Components, using the B3LYP Functional with 6-31G(d) Basis Set.

\begin{tabular}{cc}
\hline Substance & Total Energy, $\mathbf{~ k J ~ m o l}^{\mathbf{1}}$ \\
\hline KNa tartrate: urea: $\mathrm{H}_{2} \mathrm{O}(1: 2: 4)$ & -5576863 \\
\hline $\mathrm{KNa}$ tartrate & -3591820 \\
\hline Urea & -591095 \\
\hline Water & -200512 \\
\hline$\Delta \mathrm{E}_{\mathrm{NB}}$ & -826 \\
\hline
\end{tabular}

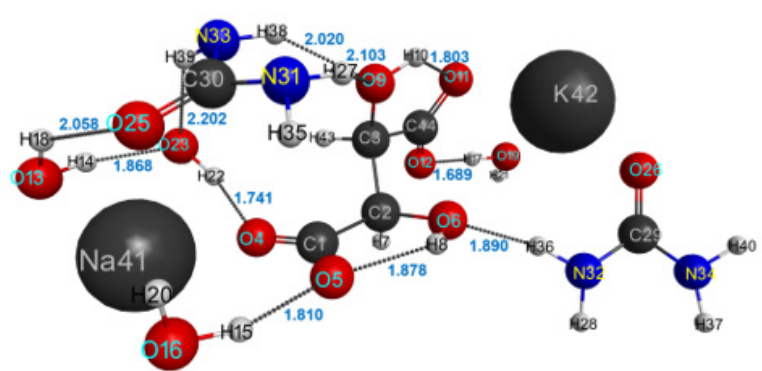

Figure 2. Optimized Structure for Adduct of $K N a$ tartrate:urea (1:2), with 4 Water Molecules, Dashed Lines Represent the H-bonding Interactions, Lengths in $A$, using the B3LYP functional with 6-31G(d) basis set.

\section{CONCLUSIONS}

Deep eutectic solvents make use of cheap, biodegradable components with little to zero toxicity, allowing these systems to be better alternatives to ionic liquids and other organic solvents in common applications. The variety of possible DES components permits modification of the physical properties of the eutectic system fit for its desired purpose. Production of DES usually involves the use of simple methods like heating and grinding, and proceeds with $100 \%$ atom economy, which makes it highly applicable for large-scale production. In this study, we have synthesized a eutectic system based on $\mathrm{KNa}$ tartrate: urea (1:2) through grinding, sonication, and heating to minimize decomposition. From the phase diagram, we have determined that the eutectic temperature is at $19.83 \pm 0.76{ }^{\circ} \mathrm{C}$, comparable to choline chloride: urea (1:2). The eutectic system is denser than water and has low viscosity. We also explored looking into the interactions present in KNa tartrate: urea (1:2) adduct through DFT calculations. We found that significant intermolecular $\mathrm{H}$-bonding interactions occur between urea hydrogens and hydroxyl oxygens of tartrate. There are also intramolecular H-bonding between the carboxylate oxygens and the hydroxyl protons. Electrostatic interactions between $\mathrm{Na}^{+}$ and $\mathrm{K}^{+}$ions and carboxylate site also exist. With a negative computed gas phase $\Delta \mathrm{E}_{\mathrm{NB}}$, these noncovalent interactions account for the stability of the KNa tartrate: urea (1:2) system. 


\section{REFERENCES}

Abbott AP, Capper G, Davies DL, Rasheed RK, Tambyrajah V. Novel solvent properties of choline chloride/urea mixtures. Chem Commun. 2003; (1):70-1.

Abbott AP, Capper G, Gray S. Design of Improved Deep Eutectic Solvents Using Hole Theory. ChemPhysChem. 2006; 7(4):803-6.

Abbott AP, Barron JC, Ryder KS, Wilson D. EutecticBased Ionic Liquids with Metal-Containing Anions and Cations. Chem - Eur J. 2007; 13(22):6495501.

Abbott AP, Harris RC, Ryder KS. Application of Hole Theory to Define Ionic Liquids by their Transport Properties. J Phys Chem B. 2007; 111(18):4910-3.

Abbott AP, Harris RC, Ryder KS, D'Agostino C, Gladden LF, Mantle MD. Glycerol eutectics as sustainable solvent systems. Green Chem. 2011; 13(1):82-90.

Bode B, Gordon MS. Macmolplt: a graphical user interface for GAMESS. J Mol Graph Model. 1998; 16:133-138.

Clarke CJ, Tu W-C, Levers O, Bröhl A, Hallett JP. Green and Sustainable Solvents in Chemical Processes. Chem Rev. 2018; 118(2):747-800.

Craveiro R, Aroso I, Flammia V, Carvalho T, Viciosa MT, Dionísio M, 'Barreiros S, Reis RL, Duarte ARC, Paiva A. Properties and thermal behavior of natural deep eutectic solvents. J Mol Liq. 2016; 215:534-40.

D'Agostino C, Harris RC, Abbott AP, Gladden LF, Mantle MD. Molecular motion and ion diffusion in choline chloride based deep eutectic solvents studied by $1 \mathrm{H}$ pulsed field gradient NMR spectroscopy. Phys Chem Chem Phys. 2011; 13(48):21383-91.

Deacutis JJ, VanSteele DR, Bailey RA, Desai SB, Hepfinger NF, Hollinger HB, et al. Experiments of Modern Chemistry: Simultaneous Recording of Multiple Cooling Curves. J Chem Ed. 1997; 74(6):732.

DDBST GmbH. Dortmund Data Bank [Internet]. Version 2018 [cited June 21, 2018]. Available from: http://www.ddbst.com.

Gallus J, Lin Q, Zumbühl A, Friess SD, Hartmann R, Meister EC. Binary Solid-Liquid Phase Diagrams of Selected Organic Compounds. A Complete
Listing of 15 Binary Phase Diagrams. J Chem Ed. 2001; 78(7):961.

Garland CW, Nibler J, Shoemaker DP. Experiments in Physical Chemistry, 8th ed. Boston, MA: McGraw-Hill Higher Education; 1996.

Gore S, Baskaran S, Koenig B. Efficient synthesis of 3,4-dihydropyrimidin-2-ones in low melting tartaric acid-urea mixtures. Green Chem. 2011; 13(4):1009-13.

Hanwell MD, Curtis DE, Lonie DC, Vandermeersch T, Zurek E, Hutchison GR. Avogadro: an advanced semantic chemical editor, visualization, and analysis platform. J Cheminformatics. 2012; 4:17.

Hao M-H. Theoretical Calculation of HydrogenBonding Strength for Drug Molecules. J Chem Theory Comput. 2006; 2(3):863-72.

Hayyan M, Mjalli FS, Hashim MA, AlNashef IM. A novel technique for separating glycerine from palm oil-based biodiesel using ionic liquids. Fuel Process Technol. 2010; 91(1):116-20.

Khandelwal S, Tailor YK, Kumar M. Deep eutectic solvents (DESs) as eco-friendly and sustainable solvent/catalyst systems in organic transformations. J Mol Liq. 2016; 215:345-86.

Krishnakumar V, Vindhya NG, Mandal BK, Nawaz Khan F-R. Green Chemical Approach: Low-Melting Mixture as a Green Solvent for Efficient Michael Addition of Homophthalimides with Chalcones. Ind Eng Chem Res. 2014; 53(26):10814-9.

Liu P, Hao J-W, Mo L-P, Zhang Z-H. Recent advances in the application of deep eutectic solvents as sustainable media as well as catalysts in organic reactions. RSC Adv. 2015; 5(60):48675-704.

Maugeri Z, Dominguez de Maria P. Novel choline-chloride-based deep-eutectic-solvents with renewable hydrogen bond donors: levulinic acid and sugar-based polyols. RSC Adv. 2012; 2(2):421-5.

Palomba T, Ciancaleoni G, Del Giacco T, Germani R, Ianni F, Tiecco M. Deep Eutectic Solvents formed by chiral components as chiral reaction media and studies of their structural properties. J Mol Liq. 2018; 262:285-94.

Schmidt MW, Baldridge KK, Boatz JA, Elbert ST, Gordon MS, Jensen JH, Koseki S, Matsunaga N, Nguyen KA, Su SJ, Windus TL, Dupuis M, Montgomery JA. General atomic and molecular electronic structure system. J Comput Chem. 1993; 
14:1347-1363.

Shahbaz K, Mjalli FS, Hashim MA, AlNashef IM.

Using Deep Eutectic Solvents for the Removal of Glycerol from Palm Oil-Based Biodiesel. J Appl Sci. 2010; 10(24):3349-54.

Shahbaz K, Baroutian S, Mjalli FS, Hashim MA, AlNashef IM. Densities of ammonium and phosphonium based deep eutectic solvents: Prediction using artificial intelligence and group contribution techniques. Thermochim Acta. 2012; 527:59-66.

Taylor R, Kennard O, Versichel W. The Geometry of the $\mathrm{N}-\mathrm{H}---\mathrm{O}=\mathrm{C}$ Hydrogen Bond. 3.* HydrogenBond Distances and Angles. Acta Cryst. 1984; B40:280-8.

Williams KR, Collins SE. The Solid-Liquid Phase Diagram Experiment: Updated for the Physical Chemistry Laboratory. J Chem Ed. 1994; 71(7):617.

Zhang S, Sun N, He X, Lu X, Zhang X. Physical Properties of Ionic Liquids: Database and Evaluation. J Phys Chem Ref Data. 2006; 35(4):1475-517.

Zhang Q, De Oliveira Vigier K, Royer S, Jerome F. Deep eutectic solvents: syntheses, properties and applications. Chem Soc Rev. 2012; 41(21):7108-46.

Zhang C, Jia Y, Wang H, Hong K. Main chemical species and molecular structure of deep eutectic solvent studied by experiments with DFT calculation: a case of choline chloride and magnesium chloride hexahydrate. J Mol Model. 2014; 20: 2374.

Zhu S, Li H, Zhu W, Jiang W, Wang C, Wu P, Zhang Q, Li H. Vibrational analysis and formation mechanism of typical deep eutectic solvents: An experimental and theoretical study. J Mol Graph Model. 2016; 68:158-75. 